\title{
Migrasomes: the knowns, the known unknowns and the unknown unknowns: a personal perspective
}

\author{
$\mathrm{Li} \mathrm{Yu}^{*}$ \\ The State Key Laboratory of Membrane Biology, Tsinghua University-Peking University Joint Centre for Life Sciences, Beijing Frontier \\ Research Center for Biological Structure, School of Life Sciences, Beijing 100084, China
}

Received July 9, 2020; accepted September 29, 2020; published online November 12, 2020

Citation: Yu, L. (2021). Migrasomes: the knowns, the known unknowns and the unknown unknowns: a personal perspective. Sci China Life Sci 64, 162-166. https://doi.org/10.1007/s11427-020-1827-8

I still remember the day. It was 2012. I was checking the transmission electron microscopy images from the previous day. Something unusual caught my eyes. A structure closely resembling an opened pomegranate stood outside a cell. In retrospect, I realized I had seen these structure before, but that particular image was unusual because there were several of these large structures outside the cell. Some of them were empty, some of them had a few vesicles inside, and some of them were packed with vesicles. Just that day, provoked by that particularly striking image, I started to wonder what these structures were. Fragments of dead cells? Too neat. Exosomes? Too big. Shedding vesicles? Maybe. If so, what are the vesicles inside? Starting from this TEM image, we began our journey to understanding the pomegranate-like structure (PLS), as it was soon called in my lab at that time.

To identify a PLS marker protein, we isolated PLSs by subcellular fractionation. We performed mass spectrometry to give us a list of candidate proteins enriched on isolated PLSs. By using GFP-tagged candidate proteins, we very soon identified proteins enriched on PLSs. Among them, Tetraspanin4 (Tspan4) stood as a good marker, as it is highly enriched in PLSs. What really surprised us is that Tspan4 also labels an extensive network of membrane tethers connected to the trailing edge of the cell, with PLSs located at

*Corresponding author (email: liyulab@tsinghua.edu.cn)

Note: The original version of this article was revised because of retrospective Open Access. the branch points or the ends of the tethers. The overall appearance is of an integrated circuit (Figure 1A). A quick literature search told us that these tethers are retraction fibers, which were first identified in 1963 (Taylor and Robbins, 1963). However, there was no mention of the PLS at all. Using GFP-tagged Tspan4, we carried out time-lapse imaging to monitor the biogenesis process of PLSs. It was soon clear to us that PLS formation is linked to cell migration. When cells migrate, retraction fibers are pulled out of the rear, and PLSs start to grow on the tips or the intersections of retraction fibers. Eventually, when the cell migrates away, the retraction fibers break and PLSs are detached from the cells. Because the formation of PLSs is dependent on migration, we re-named PLSs as migrasomes (Ma et al., 2015).

The immediate question for us was what are the functions of migrasomes? We couldn't really address this question at that stage of the study, since we didn't even know whether migrasomes existed in vivo. We circumvented this problem by instead asking what migrasomes may do. Could they be a mechanism for releasing cellular contents such as cytosolic proteins? Indeed, we found that mCherry protein can be actively transported into migrasomes and then released from cells by migrasomes, a process we named as migracytosis. Could migrasomes mediate cell-cell communication? We found that migrasomes generated by one cell can be engulfed by another cell. This implies that migrasomes at least have the potential for lateral transfer of cellular contents from one cell to another, and thus may mediate cell-cell communica- 
A

B
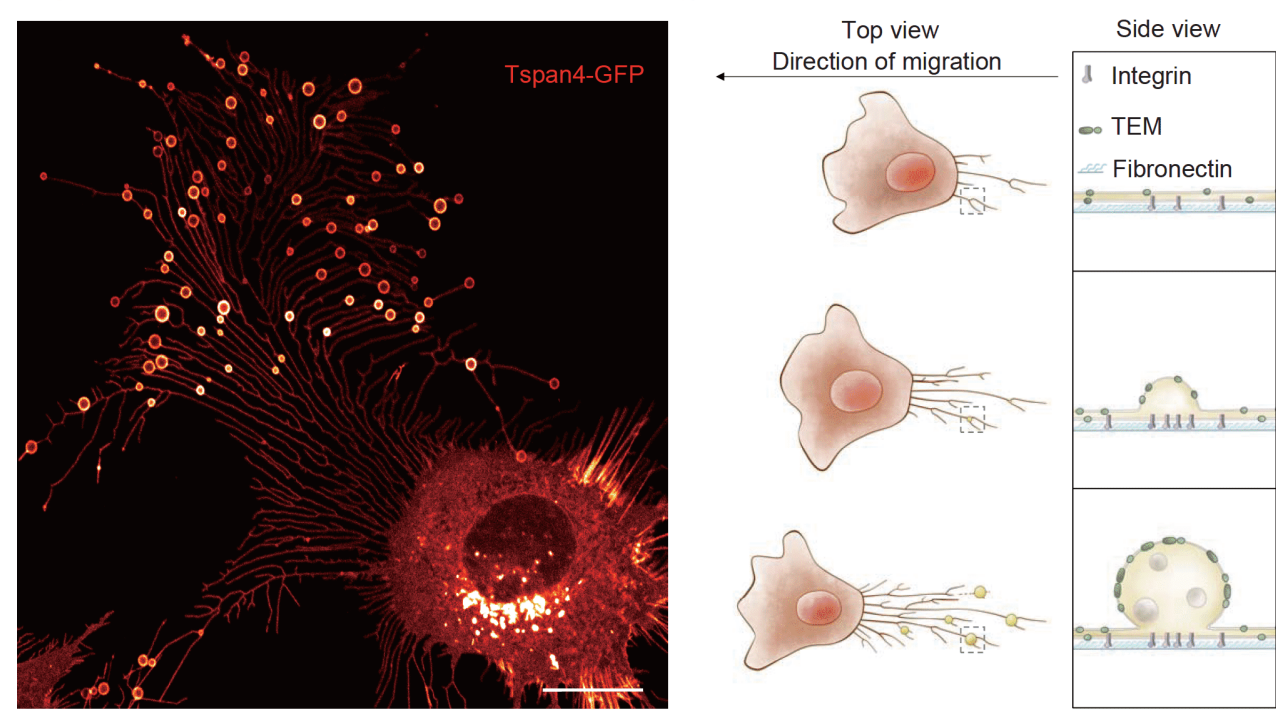

C

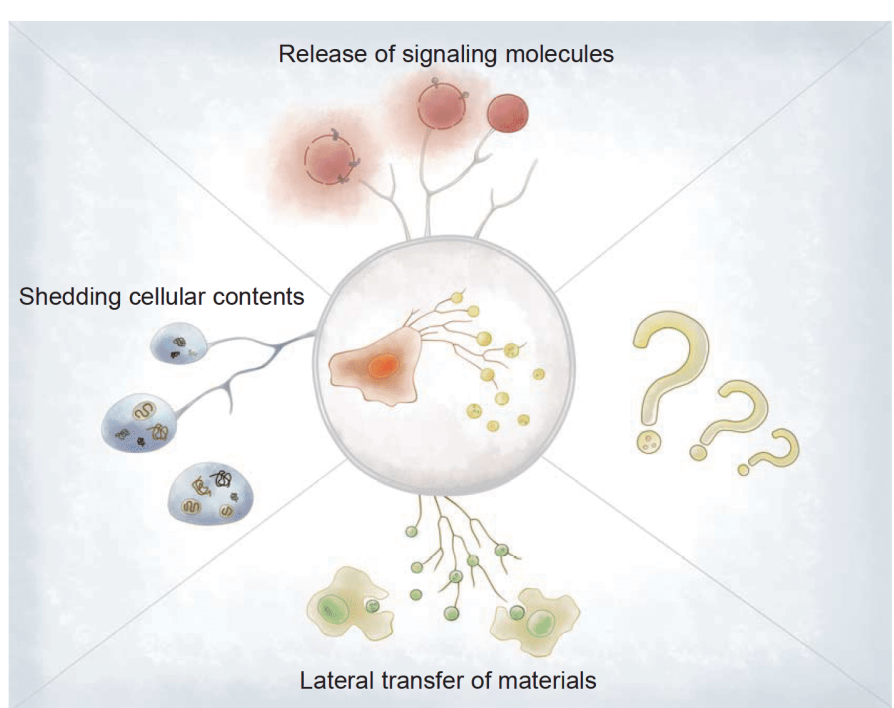

Figure 1 Migrasome, mechanism and functions. A, Migrasomes in L929 cells. Scale bar, $20 \mu \mathrm{m}$. B, Mechanism of migrasome formation. C, Functions of migrasomes.

tion. These proof-of-principle experiments do not tell us what the functions of migrasomes are. However, they do help us to formulate hypotheses which will guide the next stage of our research.

Based on these experiments, we formulated the following hypotheses. (i) Migrasomes are generated in migrating cells such as immune cells, metastatic tumor cells, and cells in developing organisms. (ii) Migrasomes are regulated by a defined pathway. (iii) Migracytosis could mediate cell-cell communication. In the past few years, our efforts to understand migrasomes have been largely guided by these hypotheses, some of which have now been validated.

Besides hypotheses, we also need a strategy. To address the question about the physiological roles of migrasomes, which is arguably the most important question, we needed to set up an in vivo model for migrasomes. Once we had the model, we could address the physiological roles of migrasome by generating migrasome-deficient animals and then checking what is wrong with these animals. To do that, of course, we first needed to know what genes are required for migrasome formation. Thus, we focused our investigation on revealing the genetic pathway regulating migrasome formation, and setting up the in vivo model for analysis of migrasomes. Along with these two goals, we also needed to develop tools for studying migrasomes, especially methods and probes to detect migrasomes.

The knowns. Regarding detection of migrasomes, we can now easily identify migrasomes by microscopy using a GFPtagged migrasome marker such as Tspan4-GFP (Ma et al., 2015). In addition, we found that wheatgerm agglutinin (WGA) stains migrasomes well, and we developed a very user-friendly protocol for detection of migrasomes using 
WGA (Chen et al., 2019).

So far, the line of research which yielded the most significant progress stemmed from our effort to understand the cellular events of migrasome formation. During migrasome formation, retraction fibers are pulled out from the trailing edge of a migrating cell. This means that retraction fibers must adhere to the extracellular matrix (ECM). Since integrins are the main molecules which adhere cells to the ECM, and our MS analysis showed that integrins are enriched on migrasomes, we focused our study on integrins. Our investigation on integrins revealed that active integrins are assembled into puncta on RFs prior to migrasome formation, and the interactions of active integrin complexes with the ECM establish the adhesion sites along the RF, which then serve as platforms for migrasome formation ( $\mathrm{Wu}$ et al., 2017).

Very soon after we identified Tspan 4 as a marker for migrasomes, we started to notice that Tspan 4 seemed to promote migrasome formation. Tspan 4 belongs to the tetraspanin protein family, which has 33 members. We found that 14 of them can promote migrasome formation when overexpressed (Huang et al., 2019). All tetraspanins have four transmembrane domains and they form so-called tetraspanin-enriched microdomains (TEMs) (Rubinstein, 2011). TEMs are about $100 \mathrm{~nm}$ in size, and are highly enriched with a set of proteins and so-called raft lipids such as cholesterol. We found that during migrasome formation, many small TEMs are assembled into micrometer-scaled macrodomains, which we named as tetraspanin-enriched macrodomains (TEMAs). Interestingly, we noticed that TEMA formation is associated with the swelling out of migrasomes from the RF, which indicates that formation of TEMAs may shape RFs into migrasomes. But how do we test this hypothesis?

The key development came by serendipity. At the beginning of this project, we planned to study the behavior of Tspan 4 on membranes using an in vitro system. The idea was to incorporate Tspan4-GFP into giant unilamellar vesicles (GUVs) and observe how Tspan4-GFP behaves on the vesicles. To do this, we first incorporate Tspan4-GFP into proteoliposomes, then we make GUVs by electrofusion of the proteoliposomes. To our surprise, upon electrofusion of Tspan4-GFP proteoliposomes (but not control proteoliposomes), we observed some beads-on-a-string-like structures, which are strikingly similar to migrasomes on retraction fibers. This observation hinted that Tspan 4 alone may be sufficient to drive migrasome formation. However, since we don't really understand what happens during electrofusion, we decided to design an in vitro system which mimics the migrasome formation in vivo.

One of the key realizations is that the pulling force generated by migrating cells may be the driving force for migrasome formation. Thus, we designed two different protocols to generate a force which can transform GUVs into membrane tethers - a process mimicking the generation of retraction fibers. In the first protocol, we stick GUVs on the button of a flow chamber, and then use liquid flow to generate a force. In the second protocol, we manually pull the membrane tether using a glass needle. In both protocols, migrasome-like structures were successfully formed on the membrane tethers which were pulled out from the Tsapn4GFP-embedded GUVs. Reconstituting migrasome formation using a chemically defined system allowed us to test the role of individual components. A clear picture soon emerged. We found that cholesterol and Tspan4 are essential for migrasome formation, and formation of migrasomes in vitro is also mediated by assembly of multiple small TEMS into micrometer-scaled TEMAs, which then swell into migrasomes.

But why does assembly of TEMAs transform a section of the membrane tether into a migrasome? The most frustrating thing in science is you did a perfect experiment, but you can't interpret the result. Fortunately, I have known Michael Kozlov from Tel Aviv university for years. He is a physicist turned biophysicist and is the leading expert on membrane shaping. Together, he and his talented graduate student Ben Zuker, quickly developed a model for the membrane shaping transformation process, which suggested that the swelling of RFs into migrasomes can automatically occur if the bending rigidity of the assembled Tspan4-enriched macrodomains is higher than the rest of the membrane. This model can be understood by the following analogy. Imagine we have a rubber band of constant thickness but with sections of different stretching rigidities. When we pull this rubber band, it will undergo stretching deformation. The stiffer sections will stretch less and will thus be thicker than the softer sections. Our model predicts that the bending modulus of the TEMA will be 5 to 10 times larger than the rest of the RF, and this was verified by directly measuring the bending modulus by atomic force microscopy (AFM). Thus, migrasome formation is driven by simple physics.

This model provides us with a framework to understand migrasome formation. More importantly, it establishes tetraspanins as the central players in migrasome formation. This knowledge enabled us to investigate the physiological roles of migrasomes (Figure 1B).

The most important and also the most difficult part of investigating the physiological roles of migrasomes and migracytosis is to establish suitable animal models. We didn't know when, where or in which animals migrasomes are present. To make the search more efficient, we formulated a few criteria for a good model. First, it must be easy to observe under a microscope. Second, the model needs to be readily amenable to genetic manipulation. Finally, we want a model which can be accessed easily - it will be great if the model comes from the lab next door. In the end, we decided to try zebrafish embryos. They are transparent and 
therefore great for microscopy study; it is easy to generate knockout fish; and, most importantly, Dr. Anming Meng, a leading developmental biologist who uses zebrafish as a model, is in the next building.

We started this project by labeling zebrafish embryos with the migrasome marker and visualizing them by live-cell imaging. In the first try, we observed abundant migrasomes and retraction fibers in the living embryos (Jiang et al., 2019). We found that migrasomes are mainly generated during gastrulation. We already knew that a set of genes, including $i t g b 1 b$, tspan $4 a$ and $t \operatorname{span} 7$, are required for migrasome formation. Therefore, we generated itgb1b, tspan $4 a$ and tspan 7 knockout (KO) zebrafish. The migrasome number was reduced in these $\mathrm{KO}$ fish, and interestingly, the fish showed organ morphogenesis defects. The most obvious phenotypes we noticed were laterality defects, including leftright reversal and bilateral duplication of various organs.

But does that mean the organ morphogenesis defects in these $\mathrm{KO}$ fish are due to reduced migrasome formation? Not necessarily, as these genes could have migrasome-independent functions which regulate organ morphogenesis. Thus, migrasome formation may have nothing to do with organ morphogenesis. This is actually a very common problem for investigating the physiological function of a particular cellular event. In many fields, this problem remains unsolvable. Fortunately for us, migrasomes can be purified, and thus we were able to carry out rescue experiments by purifying migrasomes from wild-type fish and then injecting them into the knockout embryos. The successful rescue of organ morphogenesis showed that migrasomes, rather than migrasome-independent functions of Tspan 4 and Tspan7, are indeed essential for organ morphogenesis.

But how do migrasomes regulate organ morphogenesis? We reasoned that migrasomes may contain some signaling molecules which will instruct cells during organ morphogenesis. To test our theory, we analyzed purified migrasomes using quantitative MS. Indeed, we found that a host of ligands, including chemokines, cytokines, morphogens and growth factors, are enriched in migrasomes. Among these signaling molecules, CXCL12 was particularly interesting for us, as CXCL12a knockout fish show similar laterality defects as we observed in migrasome-deficient fish. Moreover, injection of migrasomes from wild-type embryos into CXCL12a knockout embryos rescued the laterality defects. Thus, CXCL12 is the key molecule in migrasomes for regulation of laterality.

Kupffer's vesicle is a ciliated organ in the zebrafish embryo that initiates left-right development of other organs. We found that migrasomes are required for $\mathrm{KV}$ formation: in migrasome-defective embryos, KV can't form properly. KV develops from a group of migrating progenitor cells named as dorsal forerunner cells (DFCs). In migrasome-defective embryos, DFCs disperse over the course of their migration and do not reach their destination, which points to the possibility that migrasomes work as a regional chemoattractant which guides the migrating DFCs to the right position in the embryo. To test this hypothesis, we purified the migrasomes from wild-type embryos, embedded them into agarose beads, and then inserted the beads into the ventral side of embryos, where DFCs normally will not go. We found that migrasomecontaining beads can indeed attract DFCs. Thus, migrasomes are chemoattractants for DFCs.

There was still a final puzzle to solve. If migrasomes are chemoattractants for DFCs, they need to be enriched in the area which is the destination for migrating DFCs. DFCs are known to cluster on the embryonic shield; thus, we checked the areas surrounding the embryonic shield. To our surprise, we found a big cavity beneath the embryonic shield, which we named as the embryonic shield cavity. Sure enough, migrasomes are highly enriched in this cavity. Interestingly, no matter where in the embryo we inject purified migrasomes, they always end up in the embryonic shield cavity. This probably explains why our rescue experiment worked in the first place. We speculate that the "embryonic flow" generated by the movement of cells pushes migrasomes into the empty cavity, similar to the way in which floating objects are always washed to the shore at the turning point of a river.

This work established the first known physiological function of migrasomes, and more importantly, it established a paradigm for how migrasomes may work in other biological settings. In this paradigm, signaling molecules are packed into migrasomes and are released to defined locations by migrasomes, thus activating surrounding cells. In this sense, migrasomes can integrate spatial, temporal and specific chemical information which is required for coordinating the behavior of a community of cells during a complicated biological process such as embryonic development (Figure 1C). In short, a migrasome is a packet of information with a specific address for delivery.

Besides the delivery of signaling molecules, another function of migrasomes is starting to emerge. Our original work on migrasomes showed that migrasomes produced by one cell can be taken up by surrounding cells, which suggests that cellular material can be transferred laterally between cells via migrasomes. At that time, we didn't know whether this mechanism had any functional consequence. Recently, we found that migrasomes contain RNAs (Zhu et al., in press). In-depth analysis showed that the majority of RNAs enriched in migrasomes are mRNAs. This is very different from exosomes, which contain mainly short RNAs such as miRNAs. We found that Pten mRNA is highly enriched in migrasomes. When we add purified migrasomes into Ptendeficient tumor cell lines, we observed that migrasomal Pten mRNAs are translated into Pten protein in recipient cells. Eventually, the P-Akt signal is wiped out and cell proliferation is inhibited in the recipient cells. Thus, lateral 
transfer of mRNA by migrasomes can modify the recipient cells. Although at this point we don't know whether this mechanism also occurs in a more physiologically relevant setting, lateral transfer may nevertheless represent another mechanism for migrasomes to carry out their function.

The known unknowns. At this moment, there are many obvious questions which are logical extensions of our current investigation. I summarize these known unknowns in the form of questions and I share some of my thoughts regarding these questions.

(1) What is the mechanism of migrasome biogenesis? At this point, besides the few players we have identified, the pathways controlling migrasome formation are still largely unknown. Moreover, we still don't understand many basic features of migrasomes. For example, what are the intraluminal vesicles inside migrasomes? How are these vesicles sent into migrasomes? What are their functions? What factors determine the position of migrasomes along retraction fibers? What determines the number of migrasomes? Why do some cells generate many migrasomes while other cells barely generate any?

(2) What is the physiological relevance of lateral transfer by migrasomes? Our recent work showed that proteins and mRNAs can be laterally transferred to other cells via migrasomes. However, we still don't know whether this lateral transfer of material occurs in vivo and whether it has any physiological functions. Nevertheless, it is our belief that evolution does not invent something that only works in vitro.

(3) Besides embryonic development, what other biological processes are migrasomes important for? Many immune cells migrate during their development and in immune responses and they secrete a large array of signaling molecules. This sounds like a scenario in which migrasomes can play important roles. Along this line, many other biological processes, including tumor metastasis, angiogenesis, wound healing and tissue regeneration, require cell migration and secretion. We speculate that migrasomes may also play a role in these processes.

(4) Do migrasomes have cell-autonomous functions? Could cells use migrasomes as a mechanism for shedding unwanted material, which could be used for preserving homeostasis, disposing of damaged or toxic cell components, maintaining the optimal level of surface molecules...?

(5) What is the evolutionary origin of migrasomes? Did they arise from a single-celled organism in early evolution, or did they arise later after the emergence of multicellular organisms? Investigations using model systems which diversified early in the animal kingdom can help to answer this intriguing question.
Unknown unknowns. The history of science reminds us that besides known unknowns, there are unknown unknowns, which are impossible to predict based on what we already know. To confront these unknown unknowns, we must admit that nature is always more complicated than we thought. We have to be willing to constantly challenge the established paradigms, and we need to have keen eyes for observations that defy explanation in the current conceptual framework. With this mind-set and maybe with some good luck, serendipity may eventually lead us to these unknown unknowns.

Compliance and ethics The author(s) declare that they have no conflict of interest.

Acknowledgements This research was supported by The State Key Laboratory of Membrane Biology, Tsinghua University-Peking University Joint Centre for Life Sciences, Beijing Frontier Research Center for Biological Structure.

Open Access This article is licensed under a Creative Commons Attribution 4.0 International License, which permits use, sharing, adaptation, distribution and reproduction in any medium or format, as long as you give appropriate credit to the original author(s) and the source, provide a link to the Creative Commons licence, and indicate if changes were made. The images or other third party material in this article are included in the article's Creative Commons licence, unless indicated otherwise in a credit line to the material. If material is not included in the article's Creative Commons licence and your intended use is not permitted by statutory regulation or exceeds the permitted use, you will need to obtain permission directly from the copyright holder. To view a copy of this licence, visit http://creativecommons.org/licenses/by/4.0/.

\section{References}

Chen, L., Ma, L., and Yu, L. (2019). WGA is a probe for migrasomes. Cell Discov $5,13$.

Huang, Y., Zucker, B., Zhang, S., Elias, S., Zhu, Y., Chen, H., Ding, T., Li, Y., Sun, Y., Lou, J., et al. (2019). Migrasome formation is mediated by assembly of micron-scale tetraspanin macrodomains. Nat Cell Biol 21, 991-1002.

Jiang, D., Jiang, Z., Lu, D., Wang, X., Liang, H., Zhang, J., Meng, Y., Li, Y., Wu, D., Huang, Y., et al. (2019). Migrasomes provide regional cues for organ morphogenesis during zebrafish gastrulation. Nat Cell Biol 21, 966-977.

Ma, L., Li, Y., Peng, J., Wu, D., Zhao, X., Cui, Y., Chen, L., Yan, X., Du, Y., and Yu, L. (2015). Discovery of the migrasome, an organelle mediating release of cytoplasmic contents during cell migration. Cell Res 25, 24-38.

Rubinstein, E. (2011). The complexity of tetraspanins. Biochem Soc Trans $39,501-505$.

Taylor, A.C., and Robbins, E. (1963). Observations on microextensions from the surface of isolated vertebrate cells. Dev Biol 7, 660-673.

Wu, D., Xu, Y., Ding, T., Zu, Y., Yang, C., and Yu, L. (2017). Pairing of integrins with ECM proteins determines migrasome formation. Cell Res $27,1397-1400$. 